\title{
Current Topics in Saponins and the Bitter Taste
}

\author{
Guo Shuntang* \\ College of Food Science and Nutritional Engineering, China
}

*Corresponding author: Guo Shuntang, Beijing Key Laboratory of Plant Protein and Cereal Processing/ College of Food Science and Nutritional Engineering, Beijing 100083, China

Submission: 眥 March 01, 2018; Published: 笽April 26, 2018

\section{Editorial}

Saponin is a complex group of photochemical compounds widely distributed in more than 100 families of plants such as genus Saponaria, family Caryophyllaceae, genus Sapindus, family Aceraceae, hippocastanaceae, legumes, allium species, Gynostemma pentaphyllum, ginseng, red ginseng and so on. Although most saponins are plant-derived, there are additional species isolated from a few marine organisms like star fish and sea cucumber. The composition and contents of saponins vary significantly depending on the source. Historically, saponins have been regarded as the anti-nutrient factors and poisons. However, numerous researches highlighted their health benefits and the role in pharmaceutical or medical applications. Much more attention has been given to their structures, properties, novel utilizations and commercial potential. In some cases and fields, the use of saponin is deeply limited due to the bitter taste. Not only taken as medicine but also food ingredients, the saponin taken orally would be absorbed more efficiently. The tense bitterness may reduce the compliance and the acceptance of patients especially children, leading to failures to take medication properly. Taste masking or concealing the objectionable taste of active ingredients like saponin is a key issue for pharmaceutical companies, doctors and pharmacists and also food researchers.

More than 50 kinds of biological activities were reported by hundreds of articles over recent decades. In traditional Chinese medicine, saponins naturally contained in plants are mostly considered as effective medicinal ingredients such as licorice, gynostemma pentaphyllum, ginseng, pseudo-ginseng [1]. Saponins from saponaria, soybean, chick pea, alfalfa, fenugreek, quillaja, gypsohila, garlic and many other plants are proved to lower the cholesterol via the inhibition of the absorption of cholesterol from the small intestine, or the reabsorption of bile acids [2]. Anticancer ability is another health benefit of saponin according to lots of reports, particularly the ginseng saponin. Scholars indicated that the intake of sea cucumber saponin liposomes might be able to ameliorate obesity-induced inflammation and insulin resistance. Saponins have also been used as adjuvants in vaccines with the ability to impact the immune system. They would form pores in cell membrane bilayers, helping large molecules such as proteins transported through cell membranes. The most common used immunological adjuvants are quillaja saponins, and others like soyasaponins, saponaria saponins, jujubosides, quinoa, lablabosides, gypsophila also have been reported [3].

The complex structures of saponins in different species and groups result in the differences of physical, chemical, and biological properties. The solubility, stability, various bio-effects on animals or human beings talked above generally originates in the structure. Saponins are glycosides consisting of a steriod aglycone or a triterpenes backbone (sapogenin) with one or more sugar side chain attached. The concurrence of the no polar sapogenin and the water soluble sugar chain causes the ability of a saponin to foam. That is known to all and is where its name comes from. Though most saponins taste bitter in common, the sweetness of licorice is associated with its saponin [4]. So sensory properties would also displayed complexity depended on the structure.

Since the sensory science and neuroscience have make enormous progresses, the taste perception mechanism and promoting strategies come to be another meaningful research directions. The bitter taste is one of the "basic tastes", recognized and mediate perceived by 25 bitter taste receptors (TAS2Rs or T2Rs), a subfamily of G-protein-coupled receptors (GPCRs). Children have more taste buds than adults, thus they are more sensitive to bitter. Zuker (2017) and other researchers uncovered the basic logic of the connectivity between TRCs and their partner ganglion neurons, wiring of the taste system at the periphery. A large number of techniques have been developed for masking or concealing the bitter taste such as microcapsule, $\beta$-cyclodextrin covering and adding aromatics [5]. But some damages to the structure and difficulties of absorbing would be brought out during the processing. The key molecule structure of saponin and how the chemical space effects the activation of bitter taste receptors are currently unknown yet [6-9]. Computational methods in principles of computer science could be creatively used to aid in elucidating the bitter thresholds, bitter persistence, molecular recognition, ligand binding mode, the TRCs stimulation mechanism of saponins, and also aid in the design of bitterless prodrugs [1013]. Finding an appropriate way to both protect the potency of saponins and promote a patient friendly dosage form via controlled manufacturing processes would be of great progress. 


\section{References}

1. Liu J, Henkel T (2002) Traditional Chinese Medicine (TCM): Are polyphenols and saponins the key ingredients triggering biological activities? Curr Med Chem 9(15): 1483-1485.

2. Özlem GÜ, Mazza G (2007) Saponins: Properties, applications and processing. Crit Rev Food Sci Nutr 47(3): 231-258.

3. Bomford R, Stapleton M, Winsor S, Beesly JE, Jessup EA, et al. (1992) Adjuvancity and ISCOM formation by structurally diverse saponins. Vaccine 10(9): 572-577.

4. Kennelly EJ, Suttisri R, Kinghorn AD (1996) Novel sweet-tasting saponins of the cycloartane, oleanane, secodammarane, and steroidal types. In: Waller GR, Yamasaki K (Eds.), Saponins Used in Food and Agriculture. Plenum Press, New York, USA, pp. 13-24.

5. Sohi H, Sultana Y, Khar RK (2004) Taste masking technologies in oral pharmaceuticals, recent development and approaches. Drug Dev Ind Pharm 30(5): 429-448.

6. Asl, Marjan N, Hosseinzadeh H (2008) Review of pharmacological effects of glycyrrhiza sp. and its bioactive compounds. Phytotherapy Research 22(6): 709-724.

7. Di, Pizio A, Niv MY (2015) Promiscuity and selectivity of bitter molecules and their receptors. Bioorg Med Chem 23(14): 4082-4091.

8. Rafik K (2014) Prodrugs for masking the bitter taste of drugs. Application of Nanotechnology in Drug Delivery pp. 399-445.

9. Muller RE, Morris RJ (1966) Sucrose-ammoniated glycyrrhizin sweetening agent.

10. Oda K, Matsuda H, Murakami T, Katayama S, Ohgitani T, et al. (200) Adjuvant and haemolytic activities of 47 saponins derived from medicinal and food plants. Biol Chem 381(1): 67-74.

11. Sun, Hong X, Xie Y, Ye YP (2009) Advances in saponin-based adjuvants. Vaccine 27(12): 1787-1796.

12. (2015) Tribulus. WebMD.

13. Xu R (1996) Studies on bioactive saponins from Chinese medicinal plants. Adv in Exp Med Biol 404: 371-382.

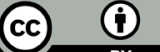

Creative Commons Attribution 4.0 International License

For possible submissions Click Here

\section{Submit Article}

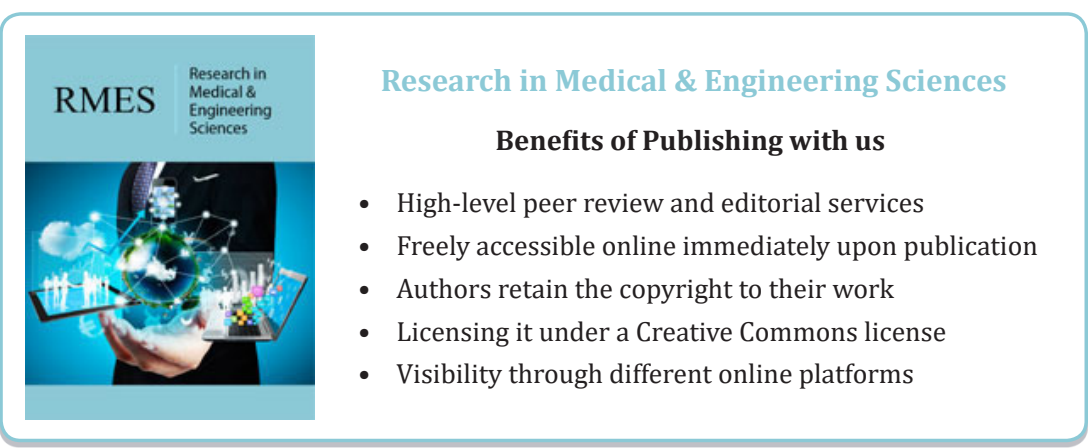

